\title{
Dual antiplatelet and anticoagulant APAC prevents experimental ischemia-reperfusion-induced acute kidney injury
}

\section{Tuuminen, Raimo}

2017

Tuuminen , R , Jouppila , A , Salvail , D , Laurent , C-E , Benoit , M-C , Syrjälä , S , Helin , H , Lemstrom , K \& Lassila , R 2017 , ' Dual antiplatelet and anticoagulant APAC prevents experimental ischemia-reperfusion-induced acute kidney injury ' , Clinical and Experimental pÿNephrology , vol. 21 , no. 3 , pp. 436445 . https://doi.org/10.1007/s10157-016-1308-2

http://hdl.handle.net/10138/237409

https://doi.org/10.1007/s10157-016-1308-2

publishedVersion

Downloaded from Helda, University of Helsinki institutional repository.

This is an electronic reprint of the original article.

This reprint may differ from the original in pagination and typographic detail.

Please cite the original version. 


\title{
Dual antiplatelet and anticoagulant APAC prevents experimental ischemia-reperfusion-induced acute kidney injury
}

\author{
Raimo Tuuminen $^{1,2} \cdot$ Annukka Jouppila $^{3} \cdot$ Dan Salvail ${ }^{4} \cdot$ Charles-E. Laurent $^{4}$. \\ Marie-Claude Benoit ${ }^{4}$ Simo Syrjälä̈ ${ }^{1,2}$ Heikki Helin ${ }^{5} \cdot$ Karl Lemström $^{1,2}$. \\ Riitta Lassila ${ }^{6,7}$
}

Received: 2 May 2016/ Accepted: 6 July 2016/Published online: 12 July 2016

(C) Japanese Society of Nephrology 2016

\begin{abstract}
Background Renal ischemia-reperfusion predisposes to acute kidney injury (AKI) and mortality. APAC, mast cell heparin proteoglycan mimetic is a potent dual antiplatelet and anticoagulant inhibiting thrombosis in several vascular models.

Methods Clinically relevant $(0.06$ and $0.13 \mathrm{mg} / \mathrm{kg})$ and high $(0.32$ and $7.3 \mathrm{mg} / \mathrm{kg})$ heparin doses of APAC and unfractionated heparin (UFH) were administered i.v. in pharmacological studies. Antithrombotic action of APAC and UFH was assessed with platelet aggregation to collagen, activated partial thromboplastin (APTT) and prothrombin (PT) times. Pharmacodynamics of $\left[{ }^{64} \mathrm{Cu}\right]$-APAC
\end{abstract}

Electronic supplementary material The online version of this article (doi:10.1007/s10157-016-1308-2) contains supplementary material, which is available to authorized users.

Riitta Lassila

riitta.lassila@hus.fi

1 Transplantation Laboratory Haartman Institute, University of Helsinki, Helsinki, Finland

2 Department of Cardiothoracic Surgery, Helsinki University Hospital, Helsinki, Finland

3 Helsinki University Hospital Research Institute, Helsinki, Finland

4 IPS Therapeutique, Sherbrooke, Canada

5 Division of Pathology, HUSLAB and Helsinki University Hospital, Helsinki, Finland

6 Coagulation Disorders Unit, University of Helsinki, Helsinki, Finland

7 Departments of Hematology and Clinical Chemistry (HUSLAB Laboratory Services), Comprehensive Cancer Center, Helsinki University Central Hospital, PoB 372, 00029 Helsinki, Finland or -UFH were monitored by PET/CT. Next, APAC and UFH doses $(0.06$ and $0.13 \mathrm{mg} / \mathrm{kg})$ were i.v. administered $10 \mathrm{~min}$ prior to renal ischemia-reperfusion injury (IRI) in rats.

Results APAC in contrast to UFH inhibited platelet aggregation. During 0.06 and $0.13 \mathrm{mg} / \mathrm{kg}$ dose regimens APTT and PT remained at baseline, but at the high APTT prolonged fourfold to sixfold. Overall bio-distribution and clearance of APAC and UFH were similar. After bilateral 30-min renal artery clamping, creatinine, urea nitrogen and neutrophil gelatinase-associated lipocalin concentrations and histopathology indicated faster renal recovery by APAC $(0.13 \mathrm{mg} / \mathrm{kg})$. APAC, unlike UFH, prevented expression of innate immune ligand hyaluronan and tubulointerstitial injury marker Kim-1. Moreover, in severe bilateral 1-h renal artery clamping, APAC $(0.13 \mathrm{mg} / \mathrm{kg})$ prevented AKI, as demonstrated both by biomarkers and survival. Compatible with kidney protection APAC reduced the circulating levels of vascular destabilizing and pro-inflammatory angiopoietin- 2 and syndecan-1. No tissue bleeding ensued.

Conclusion APAC and UFH were similarly eliminated via kidneys and liver. In contrast to UFH, APAC $(0.13 \mathrm{mg} / \mathrm{kg})$ was reno-protective in moderate and even severe IRI by attenuating vascular injury and innate immune activation.

Keywords Ischemia-reperfusion injury · Acute kidney injury · Unfractionated heparin · APAC

\section{Introduction}

Ischemia-reperfusion injury (IRI) of the kidney is the major clinical problem after aortic surgery, renal artery revascularization, and transplantation. Renal IRI is 
associated with high morbidity and mortality rates and creates an economic burden due to prolonged hospitalization and potential need for hemodialysis [1, 2]. IRI is characterized by a series of rapid glomerular and tubular damages. These predispose to deleterious short- and longterm consequences, such as acute kidney injury (AKI), and delayed function and increased immunogenicity of the allograft $[3,4]$.

Microvascular endothelial dysfunction is pivotal in kidney IRI [5]. Endothelial cell activation and barrier disruption by hypoxia and loss of vascular wall integrity predispose to thrombosis, enhanced permeability, and leukocyte adhesion [6-8]. Moreover, an extracellular matrix protein hyaluronic acid (HA) is abundant after kidney IRI [9, 10], its low molecular weight fragments acting as an endogenous danger ligand for innate immunity [11]. In experimental kidney IRI models, targeting microvascular integrity and innate immunity appears protective $[12,13]$. In clinical practice, however, the feasible strategies to limit early IRI and subsequent immunological and tissue remodeling responses remain scarce.

Anticoagulation by unfractionated heparin (UFH) is commonly administered during vascular procedures. A few studies have reported that UFH exerts limited capacity to reduce kidney IRI [14-17]. In these studies, the protective heparin effects were antithrombin mediated, however, heparin carries also anticoagulation-independent anti-inflammatory properties: inhibition of endothelial selectins, neutrophil infiltration, and complement activation. Intraoperative use of UFH has failed to protect from thrombosis in cadaveric renal allografts [18-20]. However, preoperatively initiated treatment with modified hyper-sulfated nonanticoagulant heparins reduced inflammation and chronic allograft nephropathy in experimental models of kidney IRI and allogeneic transplantation [21]. Interestingly, a multi-arm heparin conjugate coating protected hypoxic endothelial cells in vitro. These conjugates localized in the inner vessel surface in kidneys from brain-dead pigs, and reduced blood cell interactions in a vascular wound model in vivo $[22,23]$.

Semisynthetic antiplatelet and anticoagulants (APACs) are heparin proteoglycan mimics with dual antiplatelet and anticoagulant action [24, 25]. Briefly, APAC represents mast cell-derived heparin proteoglycans in the form of a semisynthetic conjugate of UFH and a protein core. In extensive in vitro and in vivo studies, we have shown that APAC is a unique locally acting antithrombotic. It specifically inhibits collagen-induced platelet aggregation, platelet deposition on injured arteries and additionally reduces fibrin formation, being an anticoagulant. In two baboon arterial thrombosis models, locally administered APAC inhibited platelet deposition to vascular injury sites or collagen surface, reduced formation or growth of thrombus and of fibrin in situ on the collagen-coated shunt [25]. Here, we studied the pharmacokinetic and pharmacodynamic profiles of APAC and UFH using labeling techniques and coagulation times, respectively. Subsequently, we examined for the first time whether intravenous pretreatment of APAC could limit a moderate 30-min and even severe 1-h bilateral kidney IRI.

\section{Methods}

First, we assessed the effects of dual antiplatelet and anticoagulant (APAC) i.v. on blood cells counts. Second, we compared APAC to UFH in platelet aggregation to collagen and coagulation times. Third, we studied pharmacokinetics based on coagulation times and bio-distribution at clinically relevant $(0.13 \mathrm{mg} / \mathrm{kg})$ and about 60 -fold supraclinical $(7.3 \mathrm{mg} / \mathrm{kg})$ dose. Fourth, we studied APAC in AKI using renal arterial clamping model.

\section{Animals}

For pharmacokinetics and bio-distribution, specific, pathogen-free, out-bred Sprague-Dawley rats (Charles River Laboratories, USA), weighing 250-300 g ( $n=4$ /group), were used IPS Therapeutique Inc., Sherbrooke, Canada. For kidney IRI male Sprague-Dawley rats (Harlan Laboratories; Horst, Nederland) weighing 250-300 g ( $n=8$ /group) were subjected to bilateral renal artery clamping. Regular rat food and tap water ad libidum were given and the rats were maintained on a 12-h light/dark cycle.

\section{Activated partial thromboplastin time and blood cell counts}

To assess activated partial thromboplastin time (APTT), rats were administered (i.v, penile vein) either with APAC (APAC2; Aplagon Oy, Helsinki, Finland) or UFH (Leo Pharma, Ballerup, Denmark) at equivalent heparin doses of $0.06,0.13$ or $0.32 \mathrm{mg} / \mathrm{kg}$ ( $n=5-8$ rats/group). At $10 \mathrm{~min}$ after sample administration, blood was drawn by cardiac puncture in $3.8 \%$ sodium citrate and rats were euthanized. To collect platelet-poor plasma (PPP) blood was centrifuged at $1200 \mathrm{~g}$ for $15 \mathrm{~min}$, and re-centrifuged at $16,100 \mathrm{~g}$ for $5 \mathrm{~min}$. APTT (Dade Actin ${ }^{\circledR}$ FSL reagent, cat no B4219-1, $\mathrm{CaCl}_{2}$, cat no ORHO37, Siemens, Munich, Germany) was measured with Sigma Amelung KC4 Delta coagulometer (Sigma Amelung, Lemgo, Germany) for the maximal recording time of $180 \mathrm{~s}$. Next, we studied the effects of APAC on blood cell counts. Rats were administered (i.v. penile vein) with APAC. Blood cell counts were determined with Sysmex KX-21 (Sysmex Corporation, Kobe, Japan). 


\section{Collagen-induced platelet aggregation}

APAC and UFH ( $3 \mu \mathrm{g} / \mathrm{ml}$; compatible with systemic heparin dosing of approximately $0.20 \mathrm{mg} / \mathrm{kg}$ ) were studied in vitro in collagen-induced platelet aggregation (Chrono-par ${ }^{\circledR}$ collagen, cat no 385 , at $2 \mu \mathrm{g} / \mathrm{ml}$, Chrono- $\log ^{\circledR}$ aggregometer, Chrono-log Corp., Havertown, PA) using platelet-rich plasma (PRP). From an anesthetized rat, blood (5-7 ml) was collected in $3.2 \%$ sodium citrate by cardiac puncture and centrifuged at $200 \mathrm{~g}, 10 \mathrm{~min}$ to obtain PRP $\left(3.5 \times 10^{8}\right.$ platelets/ml) for aggregation studies. Residual blood was centrifuged at 2000g, $10 \mathrm{~min}$ for PPP for aggregation settings.

\section{Pharmacokinetics analysis with APTT and PT}

Rats were injected with APAC and UFH at 0.13 and $7.3 \mathrm{mg} / \mathrm{kg}$ in the tail vein ( $n=4 /$ group). Blood clearance rate from the serial jugular or the contra-lateral tail vein sampling (3.2\% sodium citrate) was determined at 5, 60, and $90 \mathrm{~min}$, and at 6, 24, and $48 \mathrm{~h}$. APTT was evaluated with Actin ${ }^{\circledR}$ FSL (cat no B4219-1; $\mathrm{CaCl}_{2}$, cat no ORHO37, Siemens, Munich, Germany) and PT using Thromborel ${ }^{\circledR} \mathrm{S}$ reagents (cat no OUHP29-PK, Siemens) up to $120 \mathrm{~s}$.

\section{Bio-distribution analysis}

Male rats were injected with $\left[{ }^{64} \mathrm{Cu}\right]$-labeled 1,4,7-triazacyclononane-1,4,7-triacetic acid (NOTA)-APAC ( $n=4$ /group) and NOTA-UFH ( $n=3$ /group) at 0.13 , 0.50 , and $7.3 \mathrm{mg} / \mathrm{kg}$ in the tail vein. The distribution in kidneys, lungs, spleen, liver, and heart was followed over $48 \mathrm{~h}$ by static imaging using LabPET-8 PET/CT scanner at $1.2 \mathrm{~mm}$ resolution ( $n=2$ /group). Rats were euthanized at $48 \mathrm{~h}$ and the radioactivity in blood, kidneys, lungs, spleen, liver, heart, and muscles was measured by Cobra Gamma Counter (GMI, Inc. Ramsey, MN, USA). Data were corrected for background radioactivity and decay of ${ }^{64} \mathrm{Cu}$ (half-life of $12.7 \mathrm{~h}$ ), and the radioactivity concentration was expressed by injected dose (ID) normalized by weight of the tissue (\% ID/g).

\section{Renal artery clamping model}

The rats ( $n=8 /$ group) in a blinded set-up received 0.06 , 0.13 or $0.32 \mathrm{mg} / \mathrm{kg}$ of APAC or UFH in penile vein (i.v.) at $10 \mathrm{~min}$ before or after the onset of warm ischemia. Control rats received saline vehicle i.v. The rats were anesthetized with isoflurane $(2.5 \%)$, and a midline abdominal incision was performed. Both renal arteries were clamped either for 30 or $60 \mathrm{~min}$, depending on the study model. After clamp removal, the kidneys were inspected for recovery of blood flow and signs of internal bleeds, and the abdomen was closed. The rats were administered $1 \mathrm{ml}$ of saline and
$0.1 \mathrm{ml}$ of buprenorphine (Temgesic $0.3 \mathrm{mg} / \mathrm{ml}$, ScheringPlough, Kenilworth, NJ, USA) for postoperative maintenance of fluid balance and pain relief, respectively.

\section{Renal histology}

Semi-quantitative assessment of histological damage of renal cortex, excluding subcapsular area, was carried out by senior pathologist $(\mathrm{HH})$, as follows: 2 - $\mu$ m-thick paraffin-embedded kidney samples were stained with hematoxylin and eosin (H\&E). The severity of the parameters of tubular injury (flattening, dilatation, casts, and necrosis) was graded on $0-3$ scale: grade $0=$ no damage, grade $1=$ mild, grade $2=$ moderate, grade $3=$ severe damage, and the sum of the individual scores was presented as an overall tubular injury score $(0-12)$. The pathologist was unaware of the treatment allocation.

\section{ELISA}

Protein measurements in serum samples were performed with ELISA kits according to the protocols for angiopoietin-2 (DANG20, Quantikine-R\&D Systems, Minneapolis, MN, USA) and syndecan-1 (SEB966Ra, designed by Cloud-Clone Corp. Houston, TX, USA, assembled by Uscn Life Science Inc., Cologne, Germany). Microplate reader (Bio-Rad Laboratories, Inc., Hercules, CA, USA) was used for the analysis. Frozen $\left(-20^{\circ} \mathrm{C}\right)$ serum samples were thawed, briefly spun, and diluted 1:8 in 0.01 M PBS. Standard curves were constructed by plotting the concentration against mean absorbance and fitted with a secondorder polynomial equation for calculations.

\section{Assessment of renal function and AKI}

To analyze renal function and kidney injury, tail vein blood samples were collected under anesthesia on days 1, 2, and 3 after bilateral renal artery clamping. Serum was frozen at $-20{ }^{\circ} \mathrm{C}$ until further analysis of creatinine and urea nitrogen at the HUSLAB clinical chemistry division, Helsinki University Hospital, Helsinki, Finland. Rat neutrophil gelatinase-associated lipocalin (NGAL) was selected as a biomarker for AKI. NGAL serum levels were estimated by ELISA using mouse monoclonal anti-NGAL (ABS 039-08 from BioPorto Diagnostics A/S, Gentofte, Denmark).

\section{Tubulointerstitial injury marker, KIM-1}

For immunohistochemistry of kidney injury molecule-1 (KIM-1; AF3689 from R\&D systems, Abingdon, UK), 4- $\mu$ m-thick paraffin-embedded cross sections were stained using the peroxidase ABC method (Vectastain Elite ABC Kit, Vector Laboratories). The specimens were blocked 
for 20 min with incubation by $1.5 \%$ normal goat serum/ PBS, $\mathrm{pH} 7.4$, followed by $15 \mathrm{~h}$ with primary antibody diluted $(8 \mu \mathrm{g} / \mathrm{ml})$ with a $0.1 \%$ bovine serum albumin/ PBS at $+4{ }^{\circ} \mathrm{C}$. After PBS wash, endogenous peroxidase activity was blocked by $10 \mathrm{~min}$ with $0.1 \%$ hydrogen peroxidase $(30 \%) / \mathrm{PBS}$. The specimens were further incubated with biotinylated antibodies in PBS at RT for 30 min; detected with avidin-biotin horseradish complex in the PBS at RT for $30 \mathrm{~min}$, and monitored by 3-amino9-ethylcarbazole (AEC) substrate (Vector Laboratories). The slides were counterstained with Mayer's haemalum. To determine the density of positive cells, four random fields of each cross section quadrant were counted with $400 \times$ magnification, and scored as a sum for a $1 \mathrm{~mm}^{2}$ surface area.

\section{Danger-associated innate immune ligand hyaluronan}

Hyaluronan (HA) was stained from 4- $\mu$ m-thick paraffinembedded cross sections using a specific biotinylated bHABC hyaluronan binding complex, containing biotinylated G1 domain of aggrecan and link protein, prepared from bovine articular cartilage, detected by avidin-biotinperoxidase (Vector Laboratories; 1:200 dilution) with $0.05 \%$ 3,3'-diaminobenzidine (DAB) (Sigma Chemical Co., St. Louis, MO, USA). The staining specificity was controlled by digesting some sections with Streptomyces hyaluronidase in the presence of protease inhibitors before incubating the bHABP probe with hyaluronan oligosaccharides. Ten photographs $(400 \times$ magnification $)$ were obtained from each sample and the positive-stained area for hyaluronan was imaged computer-assisted (Zeiss Axionvision 4.4, Carl Zeiss International).

\section{Statistics}

All data are expressed as mean \pm SEM and analyzed by SPSS for Windows, version 15.0 (SPSS Inc, Chicago, IL, USA). For two-group comparison, non-parametric MannWhitney $U$ test and parametric Student's $t$ test were applied. For multiple-group comparisons, non-parametric Kruskal-Wallis test with the Dunn post hoc test and parametric ANOVA with Dunnett's correction were applied. $p<0.05$ was regarded as statistically significant.

\section{Results}

\section{APAC has antiplatelet and anticoagulant activity}

First, we assessed APTT, platelet aggregation and blood cell counts in healthy rats treated with i.v.infusion of

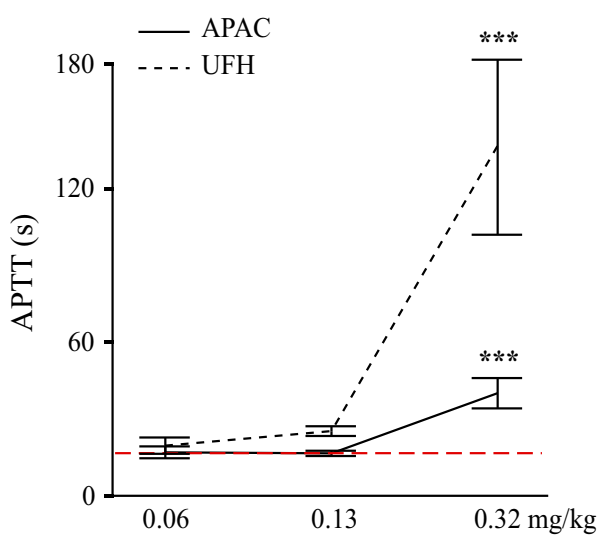

Fig. 1 Anticoagulant action of APAC and UFH. APTT (in $3.8 \%$ citrated plasma) 10 min after i.v. administration of APAC or UFH at doses of $0.06,0.13$ and $0.32 \mathrm{mg} / \mathrm{kg}$ in rats. Red dashed-line represents APTT in saline-treated rats. $n=5-8$ rats/group. $* * * p<0.001$

APAC. APTT remained at baseline at the low doses (0.06 and $0.13 \mathrm{mg} / \mathrm{kg}$ ) of APAC or UFH $10 \mathrm{~min}$ following administration (Fig. 1). High dose $(0.32 \mathrm{mg} / \mathrm{kg})$ of APAC prolonged APTT by twofold (range 16 to $40 \mathrm{~s}, p<0.001$ ), while UFH at the high dose prolonged APTT at least by fivefold (range 72 to maximal $180 \mathrm{~s}, p<0.001$ ). These APTT assessments served for the anticoagulation monitoring of the relevant dosing of APAC and UFH during the renal artery clamping.

APAC, but not UFH, inhibited collagen-induced platelet aggregation in vitro at a concentration of $3 \mu \mathrm{g} / \mathrm{ml}$, compatible with $0.2 \mathrm{mg} / \mathrm{kg}$ in vivo, APAC having maximal aggregation of $0 \%$; UFH $50 \%$; and saline $60 \%$.

Blood cell counts remained unchanged at $10 \mathrm{~min}$ after APAC administration at doses of 0.06 and $0.13 \mathrm{mg} / \mathrm{kg}$. At the high dose of $0.32 \mathrm{mg} / \mathrm{kg}$ hemoglobin was transiently reduced after APAC $(p=0.02$, Online Resource Table 1).

\section{Similar APTT-based pharmacokinetics profiles of APAC and UFH}

In APTT-based pharmacokinetic analysis of supra-clinical dose $(7.3 \mathrm{mg} / \mathrm{kg}$ ) of both APAC (range 51 to maximal $120 \mathrm{~s}$ ) and UFH (range 25 to maximal $120 \mathrm{~s}$ ) prolonged APTT during 5-90 min follow-up (Fig. 2a), without any signs of bleeding. In contrast to APTT, PT prolonged only modestly by 1.7 - to twofold during 5-60 $\mathrm{min}$, but returned to baseline at 90 min after APAC or UFH administration (Fig. 2b). Overall, these data show that APAC and UFH inhibited mainly the intrinsic pathway of coagulation, APAC markedly less than UFH. 

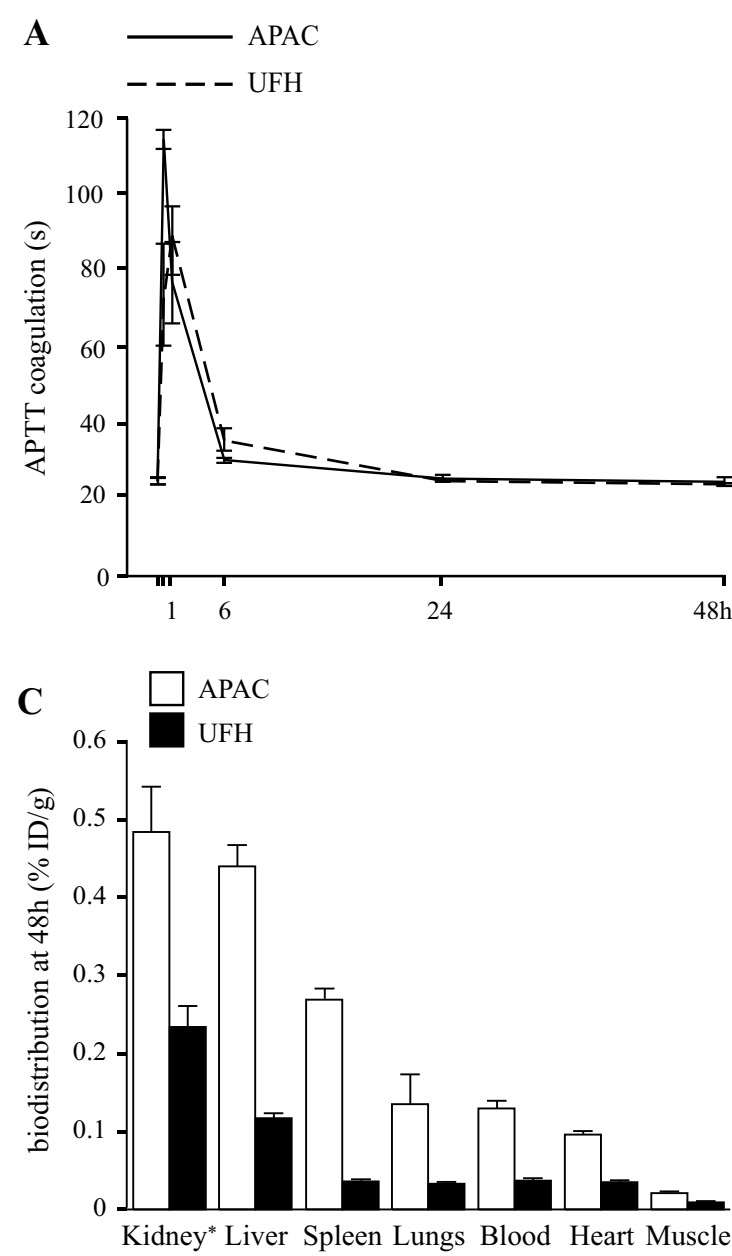

Fig. 2 Pharmacokinetics and bio-distribution of APAC and UFH by APTT and PT and by positron emission tomography (PET). APTT and PT-adjusted (in $3.2 \%$ citrated plasma) half-life of a APAC and b UFH at a supra-clinical dose of $7.3 \mathrm{mg} / \mathrm{kg}$. To analyze biodistribution by PET/CT scanning until $48 \mathrm{~h}$ the rats were given $\mathbf{c}$ [64-

\section{Bio-distribution and clearance of APAC follows heparin elimination route}

$\left[{ }^{64} \mathrm{Cu}\right]$-NOTA-APAC-based bio-distribution to kidney, liver, spleen, lungs, blood, heart, and muscle tissues when followed during $48 \mathrm{~h}$ by static imaging with PET/CT (Fig. 2c, d). After completion of the study APAC could be traced mainly in kidney, liver, and spleen (Fig. 2c, d). In the UFH-treated animals mainly kidney and secondarily liver were the sites of bio-distibution (Fig. 2c).

The exposure of $\left[{ }^{64} \mathrm{Cu}\right]$-NOTA-APAC to blood yielded the half-life $\left(\mathrm{T}^{1} / 2\right) 0.27 \mathrm{~h}$ versus UFH $0.46 \mathrm{~h}$ at the dose of $0.13 \mathrm{mg} / \mathrm{kg}$. At the dose of $0.5 \mathrm{mg} / \mathrm{kg}$ APAC T $1 / 2$ remained at $0.24 \mathrm{~h}$, whereas $7.3 \mathrm{mg} / \mathrm{kg}$ of APAC led to a $\mathrm{T}^{1 / 2}$ of $0.97 \mathrm{~h}$, while $\mathrm{T}^{1 / 2}$ was $0.38 \mathrm{~h}$ for $\mathrm{UFH}$ $(p<0.005)$. The PET scanning indicated a slower clearance and degradation of APAC in comparison to

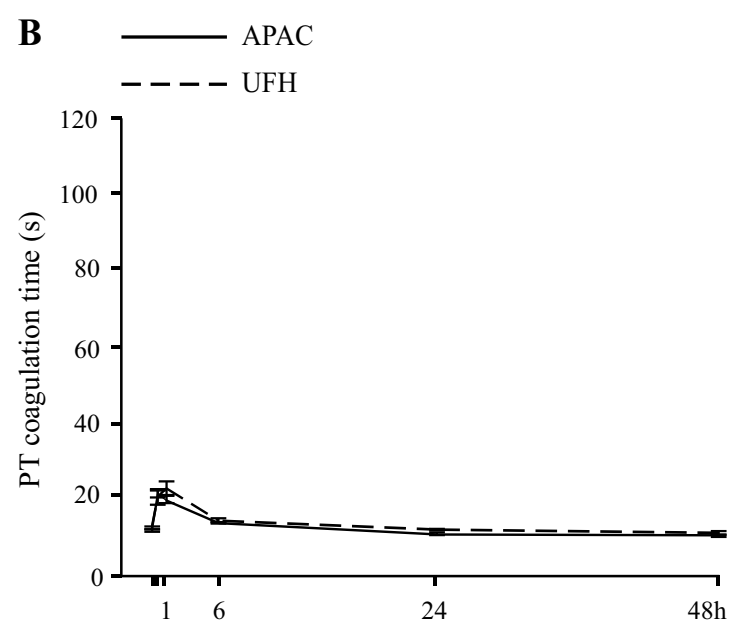

D

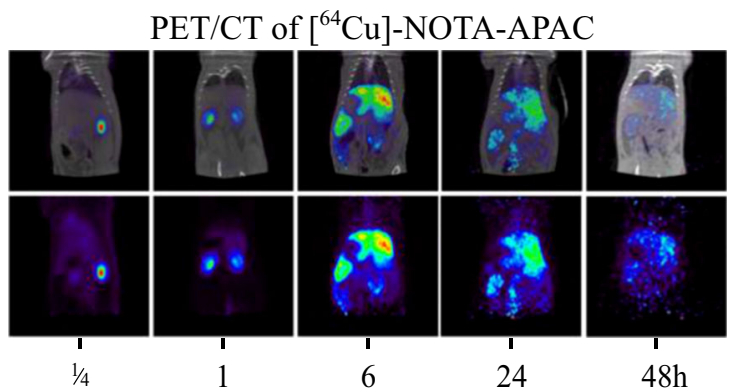

$\mathrm{Cu}$-NOTA labeled APAC. Bio-distribution at organ harvest at $48 \mathrm{~h}$ was detected with [64-Cu]-detection by gamma counter of d APAC and $\mathbf{e} U \mathrm{UFH}$ at dose of $7.3 \mathrm{mg} / \mathrm{kg}$. No signs of bleeding were observed in the animals. a, b $n=4$, c $n=2$ and $\mathbf{d}$, e $n=3-4$ rats/group. * Mean radioactivity of kidney cortex and medulla

UFH at the ultra-high dose, whereas the APTT-adjusted functional $\mathrm{T}^{1 / 2}$ of APAC was $4 \mathrm{~h}$, similar to that of UFH (Fig. 2a).

\section{APAC decreased serum NGAL levels in a 30-min kidney IRI model}

Kidney function and tubulointerstitial injury were analyzed after IRI. Clinically relevant dose range of APAC and UFH was chosen: 0.06 and $0.13 \mathrm{mg} / \mathrm{kg}$ did not prolong APTT, while APAC at an estimated $0.20 \mathrm{mg} / \mathrm{kg}$ dose inhibited collagen-induced platelet aggregation. Furthermore, the dose selection was based on the above pharmacokinetics analysis and our previous animal data [25]. The rats were randomized to either saline, UFH or APAC 10 min before 30 min clamping of both renal arteries. Serum was collected daily for 3 days after reperfusion. ELISA analysis 


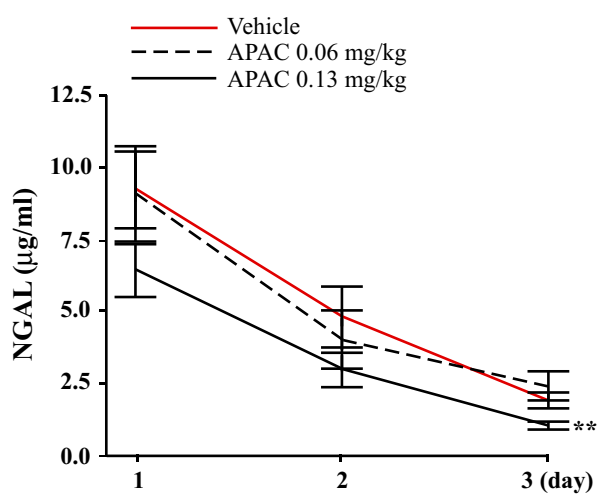

Fig. 3 Serum levels of NGAL after 30-min bilateral kidney IRI by APAC. To analyze the NGAL, serum was collected daily for 3 days from APAC 0.06 and $0.13 \mathrm{mg} / \mathrm{kg}$ i.v. pretreated rats. Control rats received saline i.v. $n=8$ rats/group. ${ }^{* *} p<0.01$

Fig. 4 Innate immune activation and histopathology of kidneys following 30-min bilateral IRI by APAC. At 3 days after reperfusion, kidney paraffin-embedded cross sections were stained for a innate immunity ligand hyaluronan, b tubulointerstitial injury marker Kim-1 and $\mathbf{c} \mathrm{H} \& \mathrm{E}$ for histopathology in APAC 0.06 and $0.13 \mathrm{mg} / \mathrm{kg}$ i.v. pretreated rats. a The area positive for hyaluronan was measured with computerassisted imaging. c $C$ epithelial casts; $D$ tubular dilatation; arrowhead epithelial flattening; arrow epithelial necrosis. Control rats received saline i.v. IgG controls in the insets. $n=8$ rats/group. $* p<0.05$
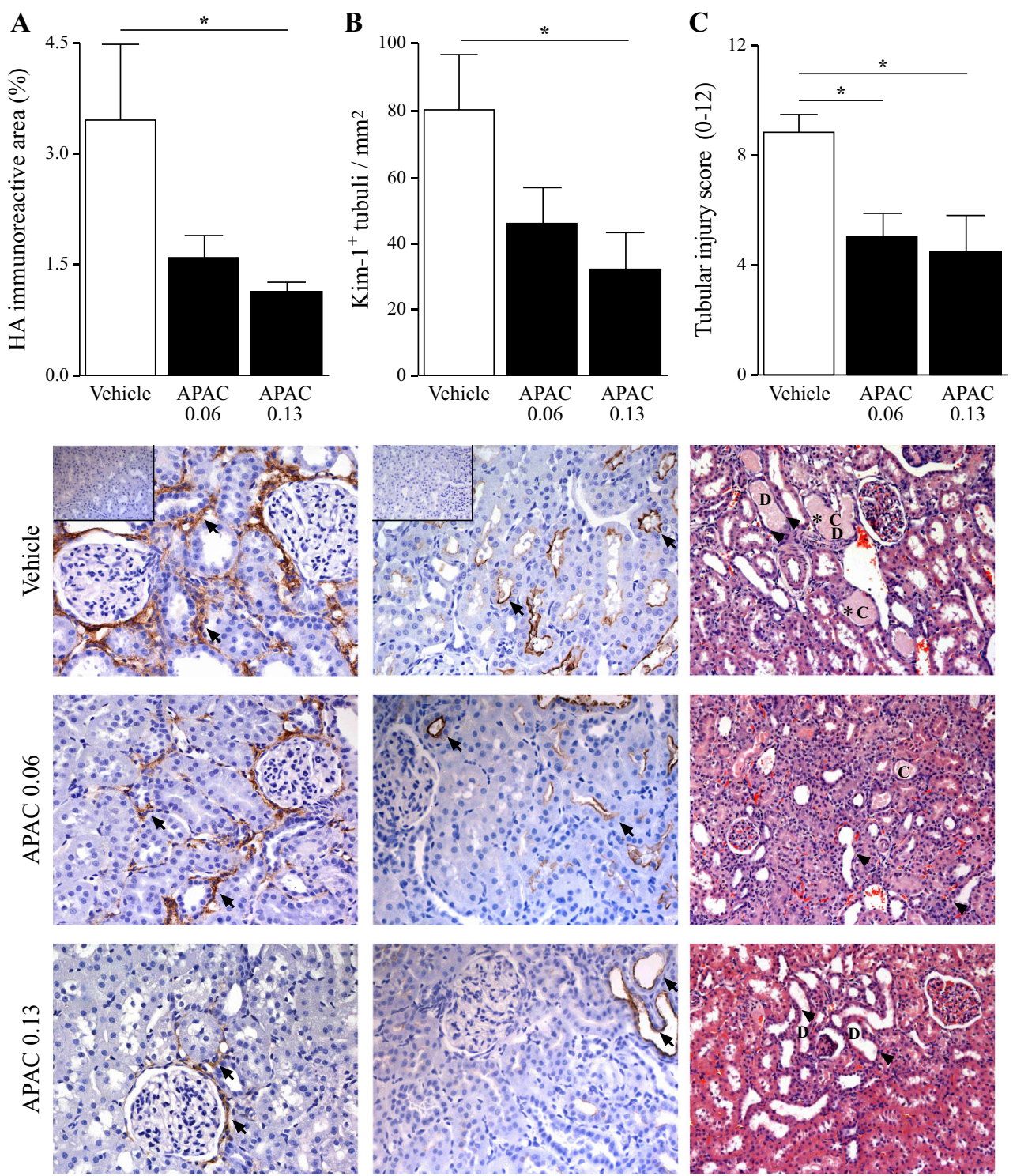

$\mathrm{HA}^{+}$area revealed that APAC $0.13 \mathrm{mg} / \mathrm{kg}$ treatment decreased serum NGAL levels ( $p<0.01$, Fig. 3).

\section{APAC decreased innate injury in 30-min kidney IRI model}

Right kidneys were collected 3 days after reperfusion for histopathological analysis. The renal cortical accumulation of innate immunity ligand, hyaluronan was reduced in kidneys of the $0.13 \mathrm{mg} / \mathrm{kg}$ APAC-treated rats compared with those of vehicle-treated animals ( $p<0.05$, Fig. 4a). Moreover, Kim-1 density implied decreased tubulointestinal injury in kidneys of APAC-treated rats compared with those of vehicle treatment ( $p<0.05$, Fig. $4 \mathrm{~b}$ ).

Semi-quantitative analysis of tubular dilatation, epithelial necrosis, flattening and casts revealed severe tubular injury 

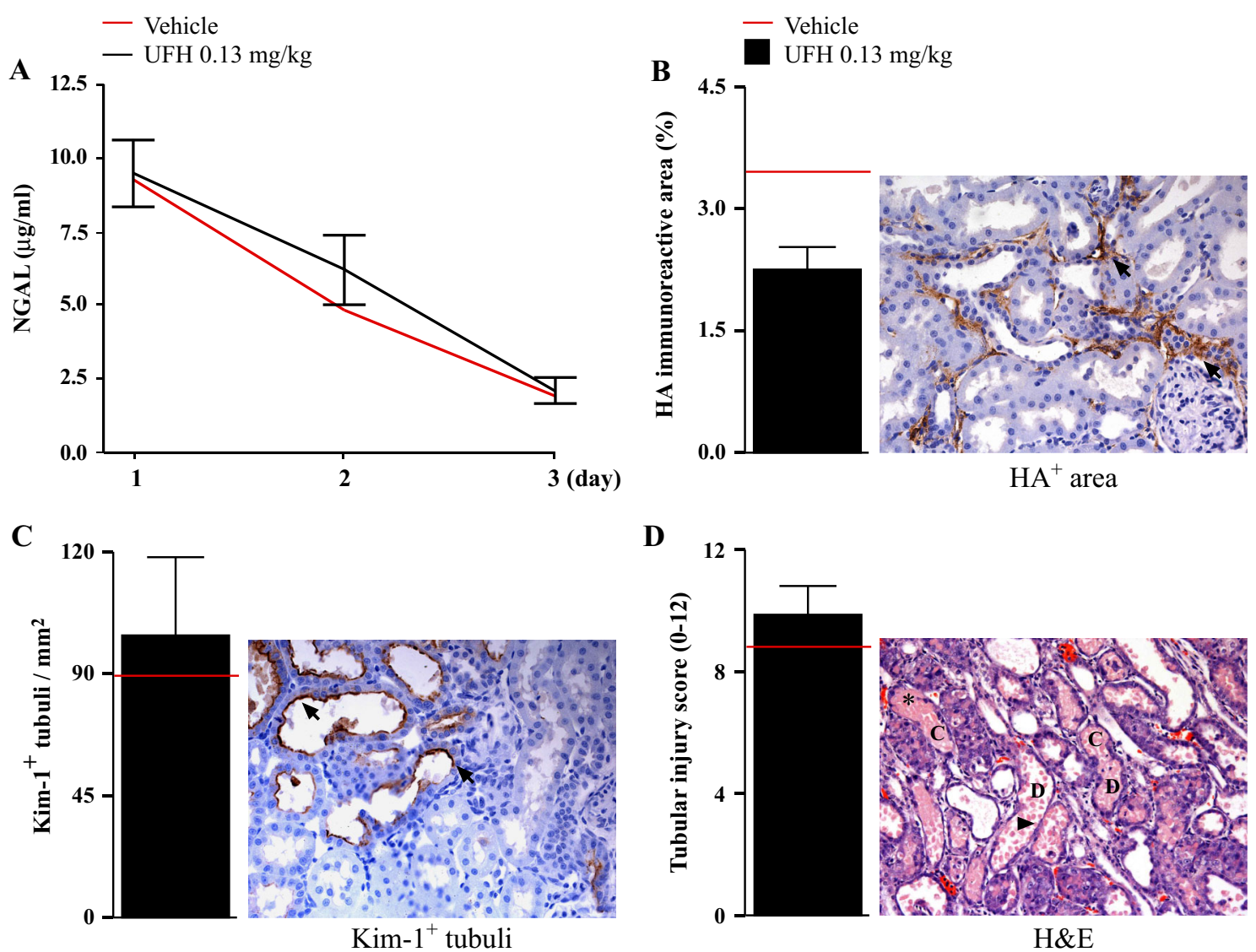

D

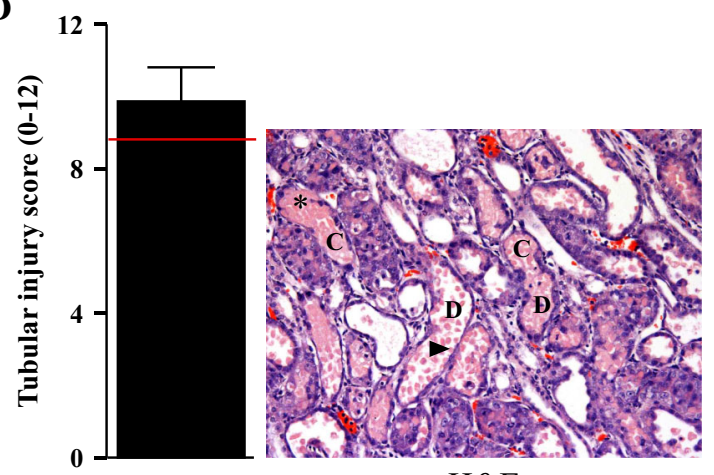

$\mathrm{H} \& \mathrm{E}$

Fig. 5 Kidney function, tubulointerstitial injury, innate immune activation, and histopathology after 30-min bilateral kidney IRI by UFH. UFH was administered at $0.13 \mathrm{mg} / \mathrm{kg}$ i.v. $10 \mathrm{~min}$ before inducing kidney IRI. To analyze kidney function and tubulointerstitial injury serum was collected daily for 3 days to assess a NGAL. Kidney paraffin-embedded cross sections were stained for $\mathbf{b}$ innate

3 days after IRI in vehicle-treated animals (Fig. 4c). Both 0.06 and $0.13 \mathrm{mg} / \mathrm{kg}$ pretreatment of APAC declined the total tubular injury score, as only mild tubular dilatation and flattening of epithelium were observed in isolated tubular cross sections of APAC-treated rats $(p<0.05$ for both, Fig. 4c). The correlation between renal cortical hyaluronan accumulation and semi-quantitative histopathological tubular injury score was significant $(r=0.43, p=0.04)$.

Neither APAC 0.06, 0.13 nor $0.32 \mathrm{mg} / \mathrm{kg}$ had an effect on blood cell count 3 days after administration, when compared with the vehicle-treated control rats (Online Resource Table 2).

\section{UFH did not decrease innate or tubulointerstitial injury in 30-min kidney IRI model}

In contrast to APAC, tubulointerstitial injury measured for 3 days following IRI was comparable between UFH $0.13 \mathrm{mg} / \mathrm{kg}$ and vehicle-treated rats with respect to NGAL immunity ligand hyaluronan, c tubulointerstitial injury marker Kim-1 and $\mathbf{d} H \& \mathrm{E}$ for histopathology. b The area positive for hyaluronan was measured with computer-assisted imaging. d $C$ epithelial casts; $D$ tubular dilatation; arrowhead epithelial flattening; arrow epithelial necrosis. Control rats received saline i.v. $n=8$ rats/group

levels (Fig. 5a). Moreover, UFH failed to protect the kidneys from the signals of innate immunity ligand hyaluronan, tubulointestinal injury marker Kim-1, and semiquantitative renal injury (Fig. 5b-d).

\section{APAC prevented endothelial angiopoietin-2 exocytosis, syndecan-1 glycocalyx shedding and AKI in 1-h kidney IRI model}

Finally, we evaluated the effects of $0.13 \mathrm{mg} / \mathrm{kg}$ APAC or saline administered $10 \mathrm{~min}$ before the induction of warm ischemia in the severe 60-min renal artery clamping model. Rat survival was $100 \%$ ( 8 of 8 ) in the APAC-treated, but $75 \%$ (6 of 8 ) in vehicle-treated group (Fig. 6a). Vascular activation and injury in APAC- and vehicle-treated rats were analyzed $24 \mathrm{~h}$ after IRI. APAC reduced serum levels of vascular destabilizing and pro-inflammatory angiopoietin-2 $(p<0.05$, Fig. 6b) and syndecan- $1 \quad(p<0.01$, Fig. 6c), when compared with vehicle-treated controls. 

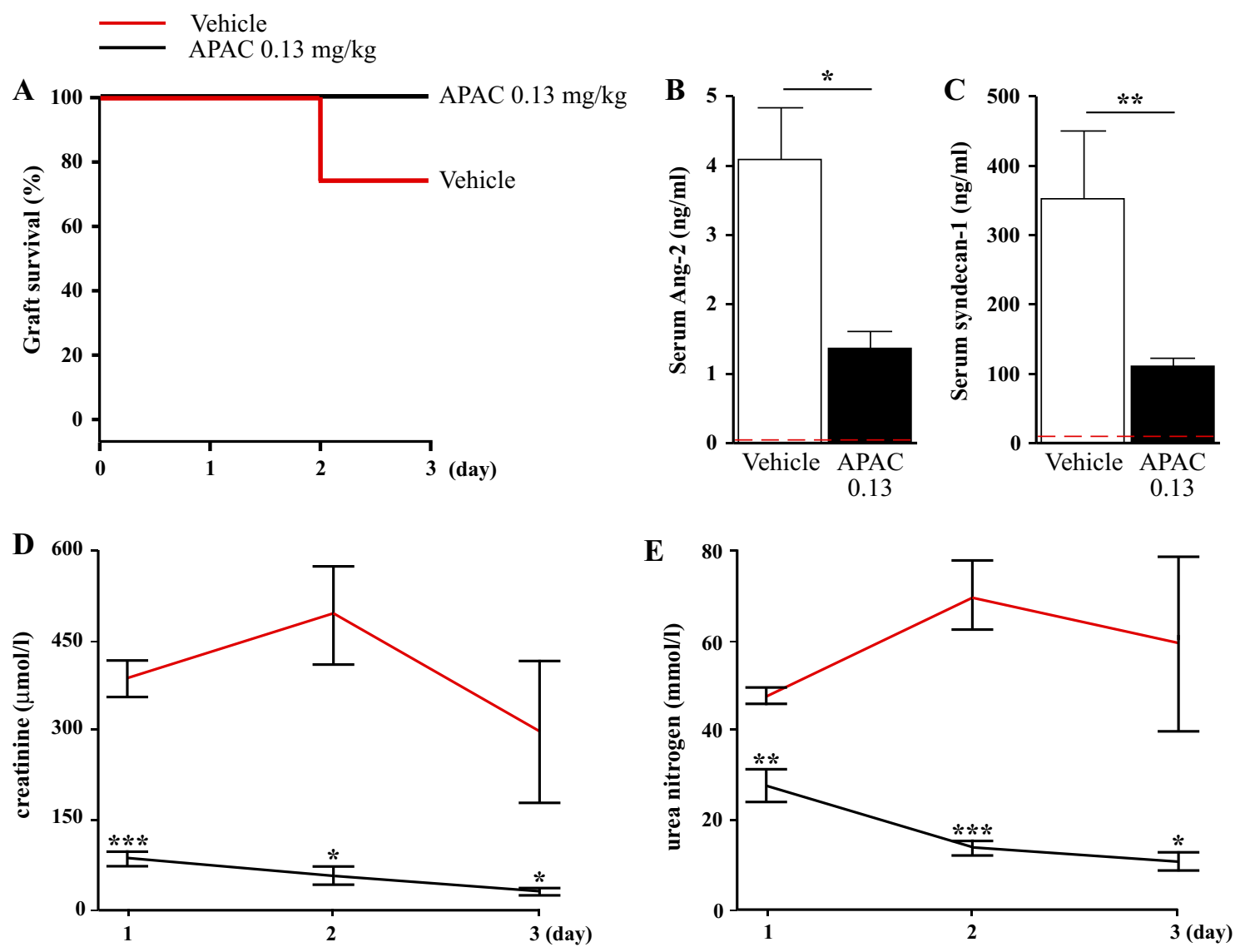

Fig. 6 Survival and serum biomarker levels of vascular injury, kidney function after severe 1-h bilateral kidney IRI by APAC. Kidneys were subjected to severe IRI in clamping both renal arteries for $1 \mathrm{~h}$, and survival (3 days), vascular injury, kidney function and rat were analyzed. Serum was collected daily for 3 days. a Rat survival,

APAC also declined creatinine $(p<0.05$, Fig. 6d) and urea nitrogen levels $(p<0.05$, Fig. 6e), when compared with vehicle-treated controls.

When administering a single i.v. dose of APAC $10 \mathrm{~min}$ after rather than 10 min prior to the 60-min kidney IRI, the beneficial effects of APAC on serum creatinine and urea nitrogen levels were lost, supporting the preventive potential of the APAC regimen (Online Resource Fig. 1).

\section{Discussion}

In this AKI model we provided evidence that APAC, a mast cell-derived heparin proteoglycan mimetic and a dual antiplatelet and an anticoagulant, importantly protects the kidneys after moderate $30 \mathrm{~min}$ and even severe $60 \mathrm{~min}$ ischemic injury. This effect was observed with APAC but not with the same dose of UFH. The reno-protection by APAC infusion prior to IRI occurred under anticoagulant conditions, assessed by APTT, which were less than measured with UFH infusion. Moreover, the systemic

b, $\mathbf{c}$ vascular destabilizing and pro-inflammatory angiopoietin- 2 and syndecan- 1 serum levels by ELISA at $24 \mathrm{~h}$ after IRI, and $\mathbf{d}$ creatinine and $\mathbf{e}$ urea nitrogen in APAC $0.13 \mathrm{mg} / \mathrm{kg}$ i.v. pretreated rats. Control rats received saline i.v. b, $\mathbf{c}$ Red dashed-line represents serum levels in native rats. $n=8$ rats/group. ${ }^{*} p<0.05,{ }^{*} p<0.01$, ${ }^{* * *} p<0.001$

effect of APAC seemed to target the renal ischemic tissue without any obvious signs of bleeding. Even raising the dose to 60 -fold concentration $(7.3 \mathrm{mg} / \mathrm{kg}$ ) did not trigger bleeds in the pharmacokinetic studies. Comparative pharmacokinetic analysis of APTT and PET imaging showed heparin-like bio-distribution and elimination routes of APAC. The local injury-associated anti-platelet properties together with anticoagulant action in the AKI model render APAC vasculoprotective after ischemic injury, but do not exclude possible anti-inflammatory mechanisms.

The important role of platelets in the initial tissue repair and vascular smooth muscle cell proliferation has been studied in vitro, and in animal models using thrombocytopenic or antibodies inhibiting platelet activation [26-32]. Depending on the activation signal, both protective and pro-inflammatory mechanisms have also been illustrated in various animal models [33]. Three important platelet-dependent mechanisms include (1) procoagulant activity and support of thrombin generation, (2) release of plateletderived PF4, P-selectin, CD40 ligand, and vascular growth factors, mediating the interactions between platelet 
activation and inflammatory response to injury, and (3) formation of neutrophil entrapment (NET), further perpetuating inflammatory injury [34]. We have previously provided both in vitro and in vivo evidence on APACs' unique dual antiplatelet and anticoagulant action [25], compatible with reduced platelet aggregation to collagen and platelet procoagulant activity. Moreover, coagulation is inhibited in a heparin-manner, i.e. thrombin time and APTT are prolonged. However, compared with UFH, APAC is more potent at decreasing thrombin-generating capacity, especially in the presence of platelets and local thrombosis, compatible with the attenuated platelet and subsequent fibrin recruitment in our earlier in vivo baboon models. We now extend these dual antiplatelet and anticoagulant observations to the avoidance of ischemiareperfusion injury, specifically to AKI. Interestingly, pretreatment with APAC reduced cortical accumulation of hyaluronan, its low molecular weight fragments being reported as a potential link between kidney IRI and innate immune activation [9-11]. APAC-without inducing tissue bleeds-reverses the functional and structural renal impairments resulting from severe warm ischemia, and maintains the overall survival of the animals. Furthermore, the systemic anticoagulant effect with the studied doses of APAC was less than that with UFH (Fig. 1). This suggests that the vasculoprotective effect reaches beyond the anticoagulation.

The adult renal microvascular network is in quiescent state and endothelium protects from thrombogenicity and leukocyte adhesion. In microvascular injury that occurs during aortic surgery, renal artery revascularization, and organ transplant, endothelial von Willebrand factor (VWF) is exposed to mediate platelet recruitment, adhesion to collagen, and subsequent procoagulant activation [35, 36]. In parallel, fibrin formation captures more platelets and leukocytes. These phenomena are regulated by endothelium via anticoagulant and platelet inhibitory mechanisms, involving heparan sulphate glycosaminoglycans and prostacyclin, nitric oxide, and ectoADPase (CD39) release [37-39]. Upon platelet activation and release, PF4 neutralizes and heparinases degrade the heparin-like molecules, fostering further coagulation and platelet activation. Additionally, the potent platelet inhibiting prostacyclin and nitric oxide are rapidly consumed under progressing thrombogenic and inflammatory microvascular conditions. When vascular permeability and endothelial injury increase the platelet contact with extracellular matrix, VWF, and collagen promote platelet accumulation, coagulation and enhance the existing inflammatory mechanisms [34].

Innate immune activation and histopathology analysis after bilateral kidney IRI revealed that APAC but not UFH was reno-protective. Moreover, our study highlights that preoperative, but not early postoperative, administration of
APAC improves kidney function in IRI. Our earlier studies in human blood and in several animal models illustrate that APACs are potent inhibitors of collagen-triggered platelets to prevent platelet deposition to both collagen and VWF [25]. Although APAC conjugates consist of heparin and show relatively compatible inhibition of thrombin and intrinsic pathway of coagulation, the inhibition of platelet aggregation and of procoagulant activity clearly distincts APAC from traditional heparins. Collagen-induced platelet aggregation was unaffected by UFH at clinical or supraclinical doses, whereas APAC dose-dependently impaired platelet aggregation and deposition on collagen surfaces $[24,25]$. Furthermore, in baboons platelet-mediated thrombosis under high shear rates was prevented by in situ administered APAC, whereas in the presence of UFH those vessels rapidly occluded. Also, in another baboon model of collagen-coated arterio-venous shunt [25], local administration of APAC reduced both platelet and fibrin deposition. In another perioperative study antithrombindependent FXa inhibitor (fondaparinux), but not UFH, improved recovery and reduced inflammation in severe kidney IRI [40]. However, fondaparinux, in contrast to heparins, is contraindicated in renal failure. In our study, decreased endothelial glycocalyx shedding of syndecan-1 and release of permeability factor Ang-2, consolidated the rapid vasculo-protective actions of APAC pre-treatment against kidney IRI.

In the future randomized clinical trials should aim at addressing whether the vasculo-protective properties of APAC, beyond regular heparin, will limit the extent and consequences of vascular injury.

Acknowledgments We gratefully acknowledge biotechnician Marja Lemponen, HUSLAB Laboratory Services, Helsinki University Hospital, for her technical assistance throughout the experiments.

\section{Compliance with ethical standards}

Ethical approval All procedures performed in studies involving animals were in accordance with the ethical standards of the institution or practice at which the studies were conducted (University of Sherbrook Ethics protocol 372-14, Canada, and the State Provincial Office in Southern Finland, ESLH-2007-07748/Ym-23).

Conflict of interest Employment: D. Salvail, C-E. Laurent and M-C. Benoit (IPS Therapeutique Inc., Sherbrooke, Canada). Stock ownership: R. Lassila, CSO and shareholder (Aplagon Oy, Helsinki, Finland). Research funding: R. Tuuminen, A. Jouppila, and S. Syrjälä (Aplagon Oy, Helsinki, Finland).

\section{References}

1. Schnitzler MA, et al. Associations of renal function at 1-year after kidney transplantation with subsequent return to dialysis, mortality, and healthcare costs. Transplantation. 2011;91(12): 1347-56. 
2. Gheorghian A, et al. The implications of acute rejection and reduced allograft function on health care expenditures in contemporary US kidney transplantation. Transplantation. 2012; 94(3):241-9.

3. McLaren AJ, et al. Delayed graft function: risk factors and the relative effects of early function and acute rejection on long-term survival in cadaveric renal transplantation. Clin Transpl. 1999;13(3):266-72.

4. Kayler LK, Srinivas TR, Schold JD. Influence of CIT-induced DGF on kidney transplant outcomes. Am J Transpl Off J Am Soc Transpl Am Soc Transpl Surg. 2011;11(12):2657-64.

5. Basile DP. The endothelial cell in ischemic acute kidney injury: implications for acute and chronic function. Kidney Int. 2007;72(2):151-6.

6. SenBanerjee $\mathrm{S}$, et al. KLF2 Is a novel transcriptional regulator of endothelial proinflammatory activation. J Exp Med. 2004; 199(10):1305-15.

7. Lin Z, et al. Kruppel-like factor 2 (KLF2) regulates endothelial thrombotic function. Circ Res. 2005;96(5):e48-57.

8. Tuuminen R, et al. Donor simvastatin treatment prevents ischemia-reperfusion and acute kidney injury by preserving microvascular barrier function. Am J Transpl Off J Am Soc Transpl Am Soc Transpl Surg. 2013;13(8):2019-34.

9. Wells A, et al. Increased hyaluronan in acutely rejecting human kidney grafts. Transplantation. 1993;55(6):1346-9.

10. Goransson V, et al. Renal hyaluronan accumulation and hyaluronan synthase expression after ischaemia-reperfusion injury in the rat. Nephrol Dial Transpl. 2004;19(4):823-30.

11. Scheibner KA, et al. Hyaluronan fragments act as an endogenous danger signal by engaging TLR2. J Immunol. 2006;177(2): 1272-81.

12. Prakash $\mathrm{J}$, et al. Inhibition of renal rho kinase attenuates ischemia/reperfusion-induced injury. J Am Soc Nephrol. 2008;19(11): 2086-97.

13. Xue $\mathrm{C}$, et al. Powerful protection against renal ischemia reperfusion injury by $\mathrm{T}$ cell-specific NF-kappaB Inhibition. Transplantation. 2014.

14. Koenig A, et al. Differential interactions of heparin and heparan sulfate glycosaminoglycans with the selectins. Implications for the use of unfractionated and low molecular weight heparins as therapeutic agents. J Clin Investig. 1998;101(4):877-89.

15. Druid H, Rammer L. Protective effect on postischemic renal edema by anticoagulation. Nephron. 1992;60(3):319-23.

16. Shin CS, et al. Heparin attenuated neutrophil infiltration but did not affect renal injury induced by ischemia reperfusion. Yonsei Med J. 1997;38(3):133-41.

17. Yagmurdur MC, et al. Antiinflammatory action of heparin via the complement system in renal ischemia-reperfusion. Transpl Proc. 2003;35(7):2566-70.

18. Bakir N, et al. Primary renal graft thrombosis. Nephrol Dial Transpl. 1996;11(1):140-7.

19. Mohan $\mathrm{P}$, et al. The role of intraoperative heparin in cyclosporine treated cadaveric renal transplant recipients. J Urol. 1999;162(3 Pt 1):682-4.

20. Nagra A, et al. The effect of heparin on graft thrombosis in pediatric renal allografts. Pediatr Nephrol. 2004;19(5):531-5.

21. Gottmann U, et al. Influence of hypersulfated and low molecular weight heparins on ischemia/reperfusion: injury and allograft rejection in rat kidneys. Transpl Int Off J Eur Soc Organ Transpl. 2007;20(6):542-9.

22. Sedigh A, et al. Modifying the vessel walls in porcine kidneys during machine perfusion. J Surg Res. 2014;191(2):455-62.
23. Nordling $\mathrm{S}$, et al. Vascular repair utilising immobilised heparin conjugate for protection against early activation of inflammation and coagulation. Thromb Haemost. 2015;113(6):1312-22.

24. Lassila R, Lindstedt K, Kovanen PT. Native macromolecular heparin proteoglycans exocytosed from stimulated rat serosal mast cells strongly inhibit platelet-collagen interactions. Arterioscler Thromb Vasc Biol. 1997;17(12):3578-87.

25. Lassila R, Jouppila A. Mast cell-derived heparin proteoglycans as a model for a local antithrombotic. Semin Thromb Hemost. 2014;40(8):837-44.

26. Reidy MA, Fingerle J, Lindner V. Factors controlling the development of arterial lesions after injury. Circulation. 1992; 86(6 Suppl):III43-46.

27. Durante W, et al. Thrombin stimulates vascular smooth muscle cell polyamine synthesis by inducing cationic amino acid transporter and ornithine decarboxylase gene expression. Circ Res. 1998;83(2):217-23.

28. Myler HA, West JL. Heparanase and platelet factor-4 induce smooth muscle cell proliferation and migration via bFGF release from the ECM. J Biochem. 2002;131(6):913-22.

29. Massberg S, et al. Activated platelets trigger an inflammatory response and enhance migration of aortic smooth muscle cells. Thromb Res. 2003;110(4):187-94.

30. Hollenbeck ST, et al. Type I collagen synergistically enhances PDGF-induced smooth muscle cell proliferation through pp60srcdependent crosstalk between the alpha2betal integrin and PDGFbeta receptor. Biochem Biophys Res Commun. 2004; 325(1):328-37.

31. Chitkara K, et al. Eptifibatide-eluting stent as an antiproliferative and antithrombotic agent: in vitro evaluation. J Invas Cardiol. 2006;18(9):417-22.

32. Myler HA, et al. Novel heparanase-inhibiting antibody reduces neointima formation. J Biochem. 2006;139(3):339-45.

33. Shi G, et al. Platelet factor 4 mediates vascular smooth muscle cell injury responses. Blood. 2013;121(21):4417-27.

34. Weyrich AS. Platelets: more than a sack of glue. Hematol Educ Prog Am Soc Hematol 2014;2014(1):400-3.

35. Siljander P, Lassila R. Studies of adhesion-dependent platelet activation: distinct roles for different participating receptors can be dissociated by proteolysis of collagen. Arterioscler Thromb Vasc Biol. 1999;19(12):3033-43.

36. Ilveskero S, Siljander P, Lassila R. Procoagulant activity on platelets adhered to collagen or plasma clot. Arterioscler Thromb Vasc Biol. 2001;21(4):628-35.

37. Chen HH, Chen TW, Lin H. Prostacyclin-induced peroxisome proliferator-activated receptor-alpha translocation attenuates NFkappaB and TNF-alpha activation after renal ischemia-reperfusion injury. Am J Physiol Renal Physiol. 2009;297(4):F1109-18.

38. Grenz A, et al. Contribution of E-NTPDase1 (CD39) to renal protection from ischemia-reperfusion injury. FASEB J Off Publ Fed Am Soc Exp Biol. 2007;21(11):2863-73.

39. Crikis S, et al. Transgenic overexpression of CD39 protects against renal ischemia-reperfusion and transplant vascular injury. Am J Transpl Off J Am Soc Transpl Am Soc Transpl Surg. 2010;10(12):2586-95.

40. Tillet $\mathrm{S}$, et al. Kidney graft outcome using an anti-Xa therapeutic strategy in an experimental model of severe ischaemia-reperfusion injury. Br J Surg. 2015;102(1):132-42. 